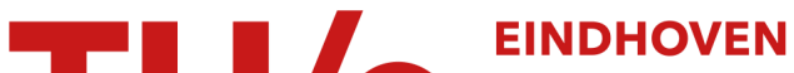 \\ UNIVERSITY OF \\ TECHNOLOGY
}

\section{Spinning of hollow porous fibres via the TIPS mechanism}

\section{Citation for published version (APA):}

Berghmans, S., Berghmans, H., \& Meijer, H. E. H. (1996). Spinning of hollow porous fibres via the TIPS mechanism. Journal of Membrane Science, 116(2), 171-189. https://doi.org/10.1016/0376-7388(96)00037-3

DOI:

10.1016/0376-7388(96)00037-3

Document status and date:

Published: 01/01/1996

\section{Document Version:}

Publisher's PDF, also known as Version of Record (includes final page, issue and volume numbers)

\section{Please check the document version of this publication:}

- A submitted manuscript is the version of the article upon submission and before peer-review. There can be important differences between the submitted version and the official published version of record. People interested in the research are advised to contact the author for the final version of the publication, or visit the $\mathrm{DOI}$ to the publisher's website.

- The final author version and the galley proof are versions of the publication after peer review.

- The final published version features the final layout of the paper including the volume, issue and page numbers.

Link to publication

\section{General rights}

Copyright and moral rights for the publications made accessible in the public portal are retained by the authors and/or other copyright owners and it is a condition of accessing publications that users recognise and abide by the legal requirements associated with these rights.

- Users may download and print one copy of any publication from the public portal for the purpose of private study or research.

- You may not further distribute the material or use it for any profit-making activity or commercial gain

- You may freely distribute the URL identifying the publication in the public portal.

If the publication is distributed under the terms of Article 25fa of the Dutch Copyright Act, indicated by the "Taverne" license above, please follow below link for the End User Agreement:

www.tue.nl/taverne

Take down policy

If you believe that this document breaches copyright please contact us at:

openaccess@tue.nl

providing details and we will investigate your claim. 


\title{
Spinning of hollow porous fibres via the TIPS mechanism
}

\author{
S. Berghmans ${ }^{a}$, H. Berghmans ${ }^{b, *}$, H.E.H. Meijer ${ }^{c}$ \\ ${ }^{a}$ K.U. Leuven, Department of Chemical Engineering, de Croylaan 46, 3001 Heverlee, Belgium \\ ${ }^{\mathrm{b}}$ K.U. Leuven, Department of Chemistry, Celestijnenlaan 200 F, 3001 Heverlee, Belgium \\ ${ }^{c}$ T.U. Eindhoven, Department of Mechanical Engineering, P.O. Box 513, 5600 MB Eindhoven, The Netherlands
}

Received 10 November 1995; accepted 15 January 1996

\begin{abstract}
Hollow porous fibres have been spun from solutions of poly(2,6-dimethyl-1,4-phenylene ether) (PPE) in cyclohexanol. This solution exhibits liquid-liquid phase separation upon cooling and two different cooling ways were used in the continuous process employed: spinning into the open air (dry spinning) and quenching in a water bath (air-gap spinning). In the first process the temperature of the spinneret was used as a parameter, while in the dry-wet spinning process the air-gap length was altered. The fibres could be used for the separation of oxygen and nitrogen and the selectivity was perfect, however the permeability was too low. In order to analyze the influence of different cooling histories across the fibre thickness, and the final local morphology, calculations were performed on cooling rates occurring in fibre spinning and specimens were prepared under a wide range of controlled cooling circumstances and analyzed using SEM. Now it is principally possible to optimize the process in order to reach asymmetric hollow fibres with an open, porous inner structure and a closed thin skin, in order to combine good selectivity with high permeability.
\end{abstract}

Keywords: TIPS; PPE; Membranes; Gas separation; Fibre spinning

\section{Introduction}

Traditionally, polymer membranes shaped as porous hollow fibres, are prepared using the diffusion induced phase separation technique or DIPS [1]. In this process, a solution of a polymer and a solvent is contacted with a non-solvent for the polymer. Because of initial interdiffusion of solvent and nonsolvent, the concentration in the polymer solution changes and, finally, phase separation occurs. Using this technique it is possible to produce asymmetric membranes with a graded pore size over the wall thickness, as well as completely symmetric mem-

\footnotetext{
${ }^{*}$ Corresponding author.
}

branes or even membranes with a closed or an open skin [2]. The selectivity of membranes is determined by the characteristics of the composing material and it does not depend on the thickness of the skin, provided that this skin is perfectly closed, since holes of course drastically decrease the selectivity [2]. On the other hand, the flux of gases through the membrane directly depends on the skin thickness and of the permeability of the supporting underlying porous structures. By decreasing the skin thickness, the gas flux increases but imperfections are more likely to occur. Therefore, generally an optimum skin thickness can be found.

Thermally induced phase separation, or TIPS, is an alternative technique used for producing porous structures [3-16]. Here a polymer is dissolved in a 
poor solvent at a high temperature. Upon cooling the homogeneous solutions, phase separation sets in, resulting in a porous material after removal (generally via extraction) of the solvent. Flat membranes were already produced using this principle, but the application to hollow porous fibres is relatively new $[17,18]$. This paper investigates the possibility to produce asymmetric fibres using the TIPS process. The system poly(2,6-dimethyl-1,4-phenylene ether) (PPE) in cyclohexanol is used, since it liquid-liquid phase separation sets in on cooling around $100^{\circ} \mathrm{C}$ and, as was already proven, fibres can be spun from solutions under these demixing conditions $[17,18]$. Depending on the cooling rate applied, two different solidification mechanisms can take place. At low cooling rates, PPE crystallizes while at high cooling rates liquid-liquid phase separation, followed by vitrification, can be realized. In the formation of hollow porous fibres, only the last mechanism is of interest, and high cooling rates are required. Two spinning techniques will be explored: dry-spinning and dry-wet, or air-gap, spinning.

\section{Experimental}

PPE with a molecular mass $\left(M_{\mathrm{w}}\right)$ of $350 \times 10^{3}$ was used. The material was supplied by TNO-KRI, The Netherlands. Solutions in cyclohexanol with a polymer concentration between 20 and $30 \mathrm{wt} \%$ PPE were used. A closely intermeshing co-rotating selfwiping twin-screw extruder (W \& P ZSK 25) was used to mix polymer and solvent [Fig. 1(a)]. The screw geometry used, is shown in Fig. 1(b). The first and largest section consists of transport elements only in order to prevent unwanted axial concentration gradients to occur, caused by hold-up of agglomerated swollen polymer particles, that are present in the early stages of the dissolution process. The total content of the extruder, which is operated completely filled, is about $360 \mathrm{~g}$ and by choosing a prescribed flow rate of the booster pump [gear pump 1 in Fig. 1(a)], the residence time in the extruder, available for complete dissolution, can be controlled. The sum of the feed rates of polymer and solvent into the extruder has to be set equal to the flow rate of gear pump 1, in order to reach a stationary situation in the spinning line. The second gear pump [gear pump 2, Fig. 1(a)] determines the throughput through the spinneret. Since the flow rate through the first gear pump (typically about $25 \mathrm{~g} / \mathrm{min}$ ) is usually too high for the spinneret which contains only one spinhole, a bypass is used in between the two pumps. The spinneret is designed by TNO-KRI Delft (Fig. 2 ), and it contains a hollow needle through which non-solvent is pumped (in case of DIPS) or air is blown (in case of TIPS) to keep the fibre open in its centre.

In dry spinning, the fibre is allowed to cool into the open air by convection and solvent evaporation,

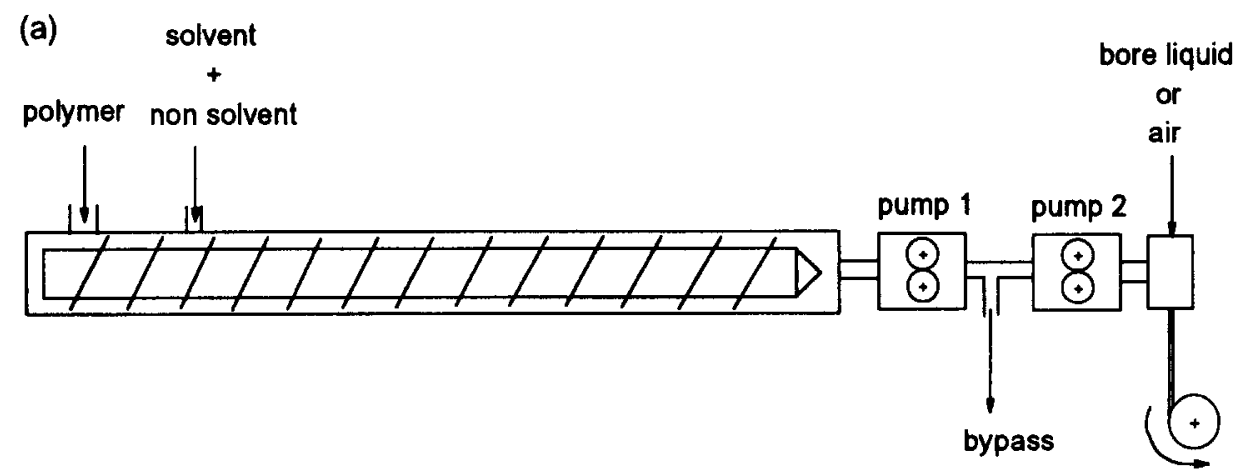

(b)

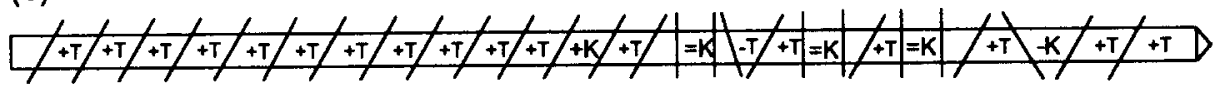

Fig. 1. (a) Schematic representation of the spinning process. (b) Screw geometry: $+T=$ positive transport, $-T=$ negative transport, $+\mathrm{K}=$ positive kneading, $-\mathrm{K}=$ negative kneading and $=\mathrm{K}=$ neutral kneading. 


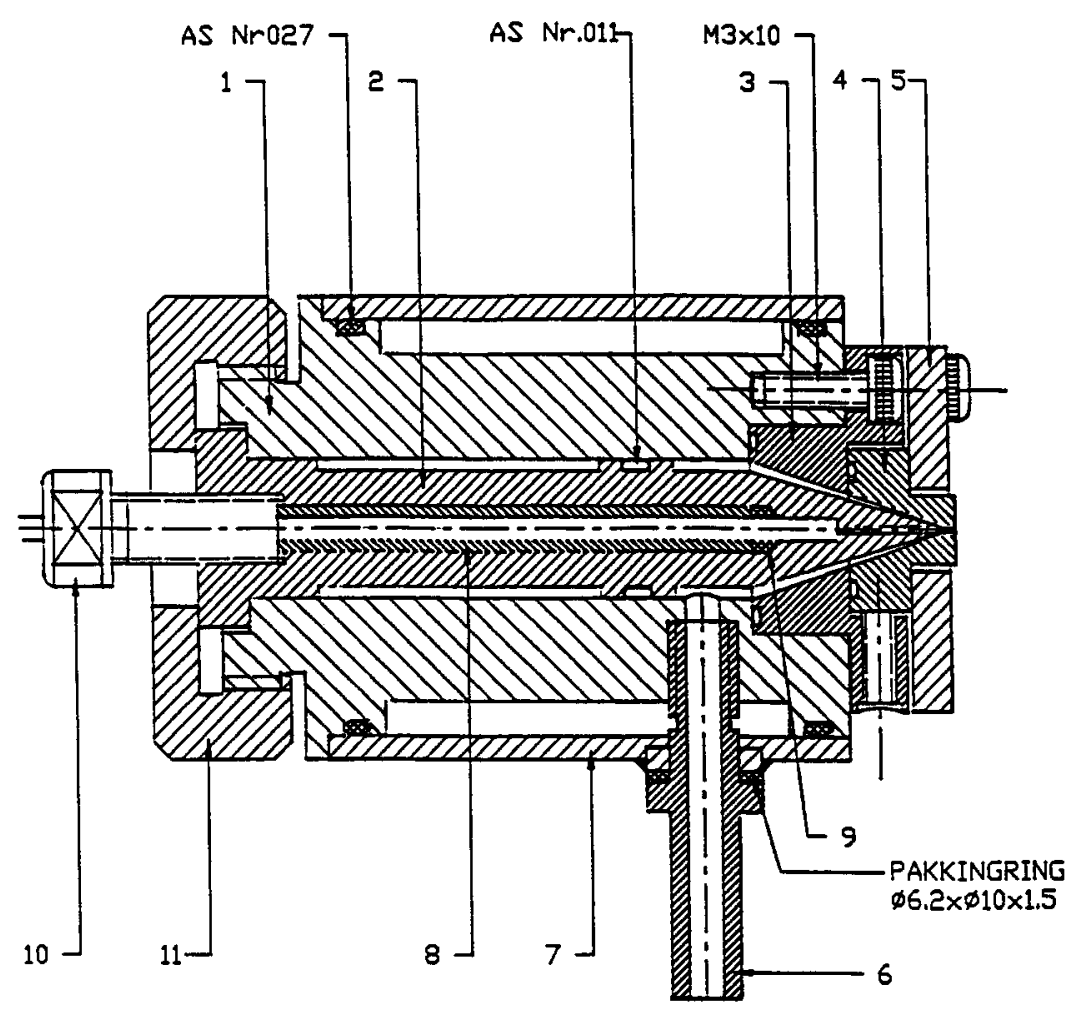

Fig. 2. Drawing of the spinneret used for spinning of hollow porous fibres, $6=$ entrance for the polymer solution, $10=$ entrance for the bore liquid or air, $4=$ exit for the fibre.

and - in the preliminary set-up used - led onto a belt conveyor which supports the fibre during transport and further cooling [Fig. 3(a)]. In the dry-wet or air-gap method, a water bath is used [Fig. 3(b)]. Water only serves as a cooling medium, and does not induce phase separation by diffusion, as is the case in the DIPS-process, since water and cyclohexanol are no mutual solvents. Cyclohexanol is hygroscopic, but at high temperatures (above $100^{\circ} \mathrm{C}$ ) water evaporates, while below $100^{\circ} \mathrm{C}$ phase separation in the polymer solution sets in, thereby also rendering it more difficult for water to dissolve. When the dry spinning method is applied, the temperature of the spinneret was varied. In the dry-wet spinning process, the air-gap was altered. Finally the fibres are washed in isopropanol to extract the remaining solvent for at least $4 \mathrm{~h}$. Subsequently the fibres were broken in liquid nitrogen and analyzed using SEM.

The fibres were tested on their gas separation properties. When the skin of the membrane is the only resistance for gas flow, the solution-diffusion mechanism determines the flux. The permeability $(1 / \mathrm{min} / \mathrm{bar} / \mathrm{m})$ is defined as [19]:

$P=D S$

where $D$ is the diffusion coefficient of the gas in the membrane material, and $S$ its solubility. Both are material parameters, therefore the permeability for a specific gas is a material parameter (in contrast, when the resistance of the porous sublayer dominates, Poiseuille or Knudsen flow through the pores determines the separation and flux characteristics).

Oxygen and nitrogen pressure is applied to the fibres and the resulting flux $J\left(1 / \mathrm{min} / \mathrm{m}^{2}\right)$ through the fibres is measured. This flux can be converted to the permeability by defining:

$P=\frac{J d}{\Delta p}$

where $d$ is the membrane thickness and $\Delta p$ the 
(a)

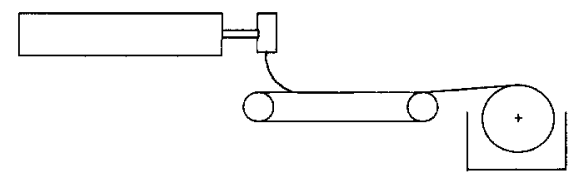

(b)

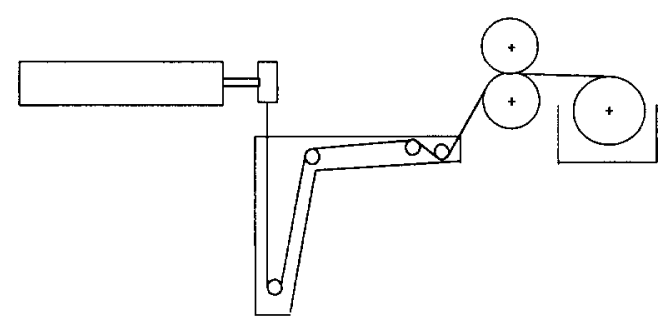

Fig. 3. Schematic representation of the two spinning processes used: (a) dry spinning and (b) air-gap spinning.

pressure difference. Usually $d$ is not known, since it represents the effective thickness of the skin, that is difficult to measure in most cases. Therefore, the reduced permeability $P / d\left(1 / \mathrm{min} . / \mathrm{bar} / \mathrm{m}^{2}\right)$ is usually used. The ratio of the permeabilities towards oxygen and nitrogen, gives a measure of the selectivity $\alpha_{\mathrm{O}_{2} / \mathrm{N}_{2}}$ of the fibre ( $d$ is the same for both cases):

$\alpha_{\mathrm{O}_{2} / \mathrm{N}_{2}}=\frac{P_{\mathrm{O}_{2}}}{P_{\mathrm{N}_{2}}}$

Since $P$ is a material parameter, each membrane material has its intrinsic selectivity for a certain gas pair. This number gives an idea of the ideal selectivity of the resulting membrane. For PPE the theoretically expected maximum $\alpha$-value for oxygen and nitrogen is 5.0 , if the separation is achieved by the solution diffusion mechanism, and it is 0.9 if Poiseuille or Knudsen flow are the main separation mechanisms [20].

As a calibration tool, morphologies were produced at controlled cooling rates. Porous samples were prepared under stationary conditions by cooling solutions with a polymer content of $20 \mathrm{wt} \%$ in DSC sample pans from 160 to $0^{\circ} \mathrm{C}$ at cooling rates between 1 and $200^{\circ} \mathrm{C} / \mathrm{min}$ (the maximum cooling rate that could be reached in the DSC). Higher cooling rates were realized using the method worked out at the chemical engineering department of the University of Palermo (Professor Stefano Piccarolo) [21,22]. In contrast to the DSC method, the cooling rates are no longer constant but vary with temperature, decreasing as the temperature goes down. Consequently, in order to define a cooling rate, reference temperature has to be chosen. We took $70^{\circ} \mathrm{C}$ as a representative temperature. The cooling rates varied between $5^{\circ} \mathrm{C} / \mathrm{s}$ and $960^{\circ} \mathrm{C} / \mathrm{s}$ or $300^{\circ} \mathrm{C} / \mathrm{min}$ and $57600^{\circ} \mathrm{C} / \mathrm{min}$. The samples were also washed in isopropanol, broken in liquid nitrogen and analyzed using SEM.

\section{Results and discussion}

\subsection{Phase behaviour of the system PPE-cyclo- hexanol}

The phase behaviour of this system has been discussed in detail in a previous paper [17]. Only the most important aspects will be recapitulated here. The temperature-concentration diagram is represented in Fig. 4(a). In this graph the melting and crystallization temperature are shown, together with the glass transition temperature. For the concentration range up to $60 \mathrm{wt} \% \mathrm{PPE}$, the crystallization temperature could only be measured at low cooling rates $\left(<20^{\circ} \mathrm{C} / \mathrm{min}\right)$, whereas the $T_{\mathrm{g}}$ in the same concentration range could only be measured at higher cooling rates. As shown in the previous paper [17], the fact that the $T_{\mathrm{g}}$-line is flat, points to the existence of a liquid-liquid phase separation domain. This is shown schematically in Fig. 4(b). This flocculation curve (dotted line) could, however, not be measured directly. The behaviour in the concentration region, important for this work, will be discussed.

Slow cooling results in crystallization of the polymer around $100^{\circ} \mathrm{C}$. The glass transition is situated at $70^{\circ} \mathrm{C}$, and is constant in this concentration region due to the presence of the liquid-liquid phase separation domain [17]. This has important consequences for the spinning process, since, after dissolution, temperatures below $70^{\circ} \mathrm{C}$ are not permitted anywhere inside the extruder or the spinneret, because in that case the solution would vitrify thereby blocking the extruder or spinneret. The temperature should however also never be below $100^{\circ} \mathrm{C}$, since crystallization would be induced, when the solution is slowly cooled [17]. Once accidentally crystallization has occurred, somewhere around $100^{\circ} \mathrm{C}$, the solution has to be heated 

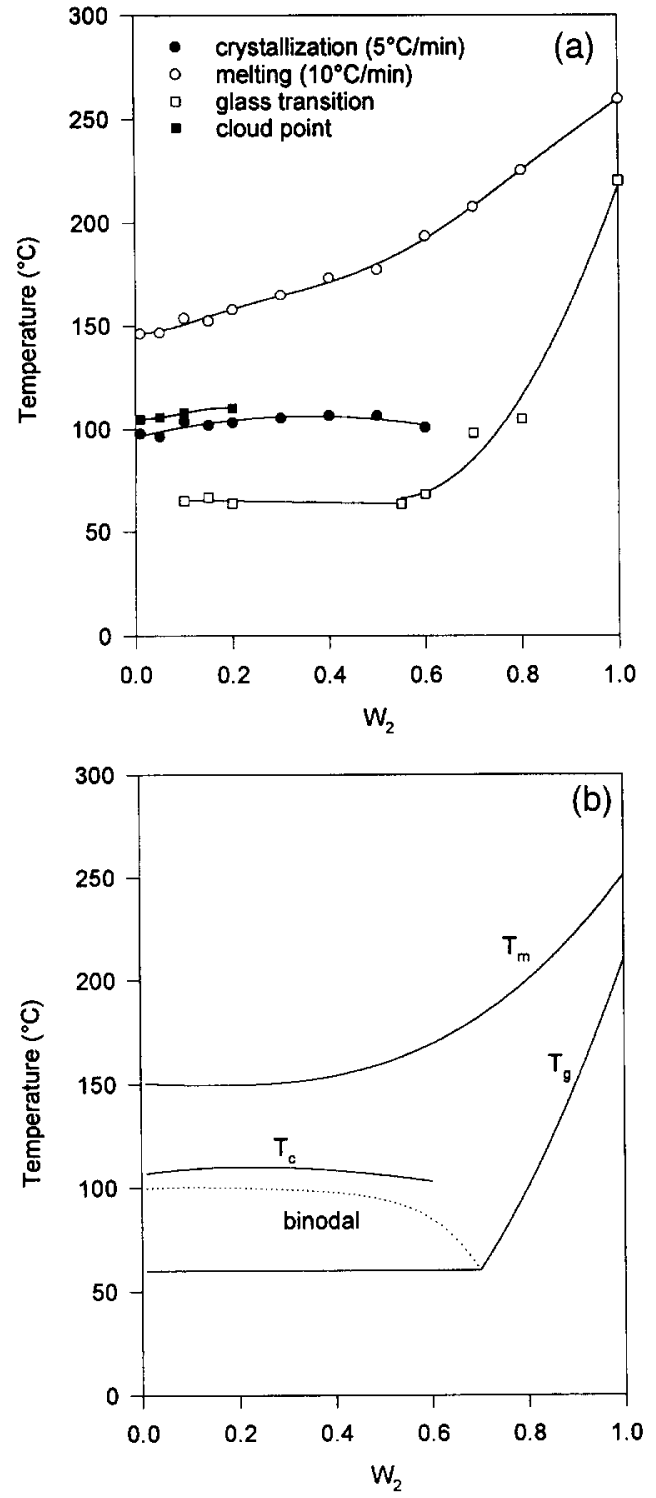

Fig. 4. (a) Phase diagram for the system PPE-cyclohexanol. (b) Schematic representation of the phase diagram.

above the melting temperature of about $150^{\circ} \mathrm{C}$. For that reason, the temperature inside the extruder is set at $150^{\circ} \mathrm{C}$.

\subsection{Dry spinning}

Fig. 5 shows cross sections and side views of fibres $(20 \mathrm{wt} \%)$ obtained via the dry spinning pro- cess. Fibre spinning was performed for temperatures of the spinneret between 150 and $80^{\circ} \mathrm{C}$. Because of the kinetics of the phase separation process, temperatures below the transition temperature are possible in the spinneret, without solidification to occur, provided that the flow rate is high enough. All fibres posses a porous wall, with a uniform pore size, and a thin skin with a rather irregular shape. The main reason for the difference in diameter between the fibres, is the difference in air flow through the needle, which was adjusted only such that the fibres did not collapse. Very accurate control revealed to be difficult in the simple preliminary set-up used. Especially at the highest temperatures, care had to be taken not inflate the fibres up to breakage, because of the extremely low viscosities of the solution.

Spinning regularly stopped due to fibre breakage. By lowering the temperature of the spinneret, the viscosity increased and the solution became much easier to spin. With the polymer molecular weight and concentrations used, at all temperatures the solution proved to be still rather fluid, when leaving the spinneret, and the fibres formed were allowed to freely flow down under gravity. The belt conveyor did not exert any noticeable draw down on the fibre.

Fig. 5 also shows side views of the outside of the fibres, which appear to be closed. At high spinning temperatures the walls show regular "stripes". At decreasing temperature, the outside of the fibre becomes less regular, and at the lowest temperature $\left(80^{\circ} \mathrm{C}\right)$ even openings in the wall are found. Irregular stripes at low spinning temperatures are more often found in dry spinning and are supposed to be caused by shrinkage due to evaporation of the solvent $[23,24]$, while the more regular stripes found at high spinning temperatures seem to be caused by the flow and not so much by shrinkage, since they extend along the whole length of the fibre.

Fig. 6 shows the gas separation properties of these fibres. The permeability for both oxygen and nitrogen increases drastically when the temperature of the spinneret is decreased. The selectivity on the other hand is low in all cases and decreases to the value of one, because of the presence of holes. In all cases separation is achieved by the solution diffusion mechanism since the selectivity is greater than 0.9 , which is the intrinsic selectivity when the resistance of the sublayer dominates [20]. If pores are present, 
they seem to be too large for Poiseuille or Knudsen flow to be important. The fibre spun at $150^{\circ} \mathrm{C}$ has a selectivity of about 2.5 , which is acceptable but not
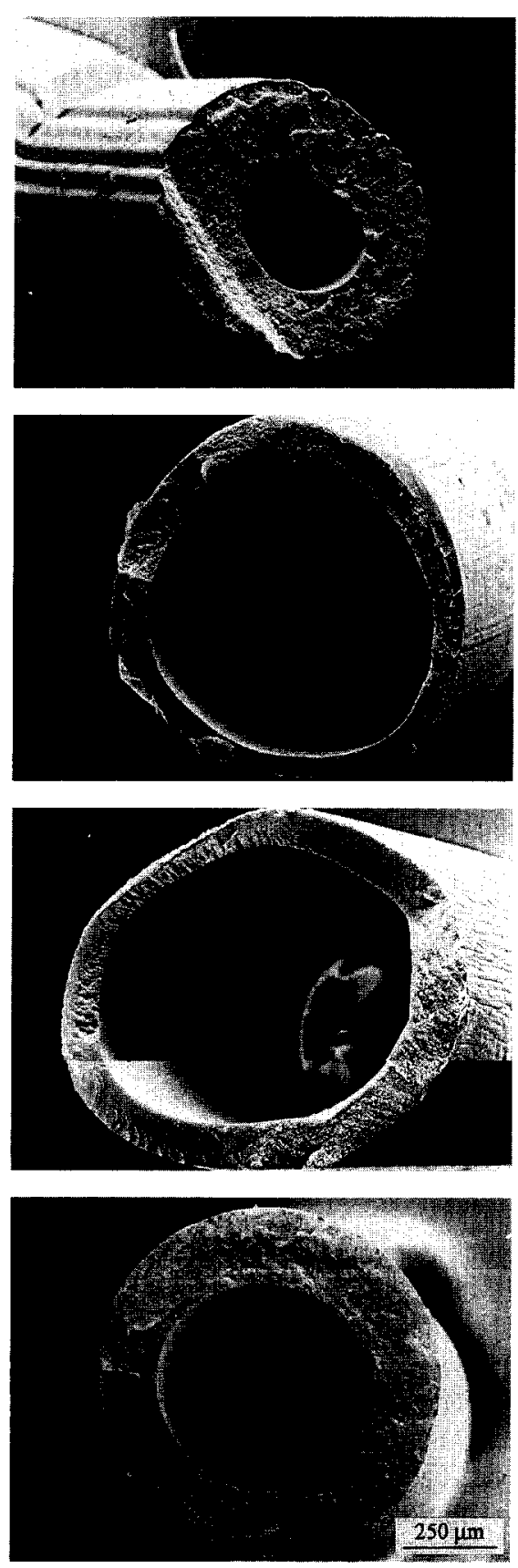

very high. The reduced permeability is, however, very low. The rate of solvent evaporation increases as the spinneret temperature is increased. This results

(a)

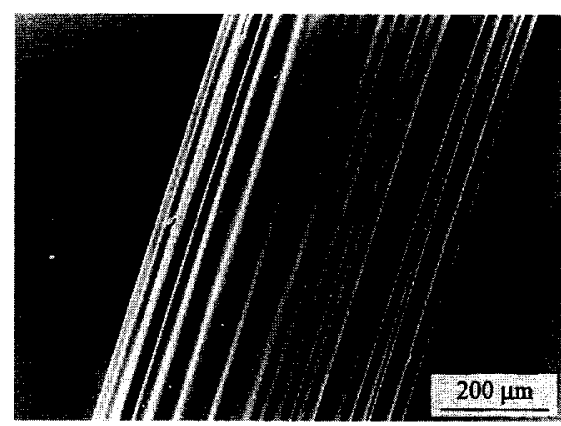

(b)

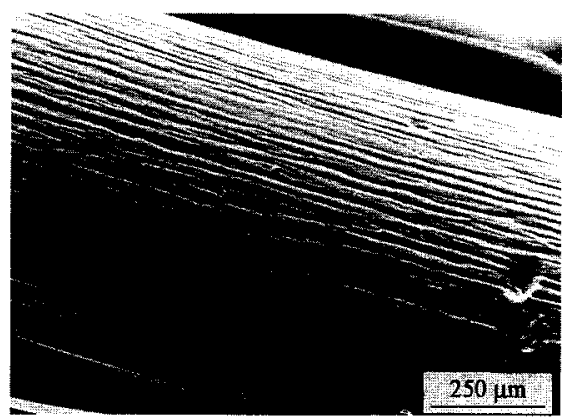

(c)

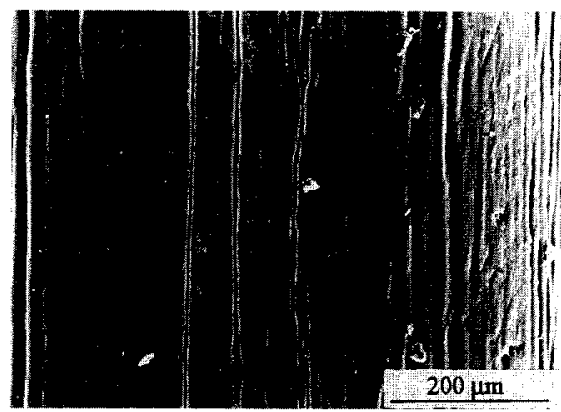

(d)

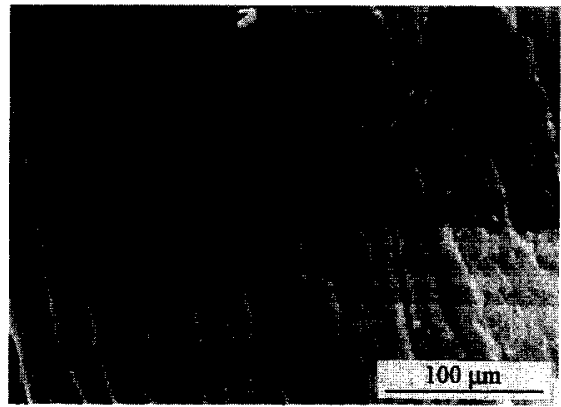

Fig. 5. Cross sections and side views of $20 \mathrm{wt} \% \mathrm{PPE}$, dry-spun fibres, for different spinneret temperatures: (a) $140^{\circ} \mathrm{C}$, (b) $120^{\circ} \mathrm{C}$, (c) $100^{\circ} \mathrm{C}$ and (d) $80^{\circ} \mathrm{C}$. 


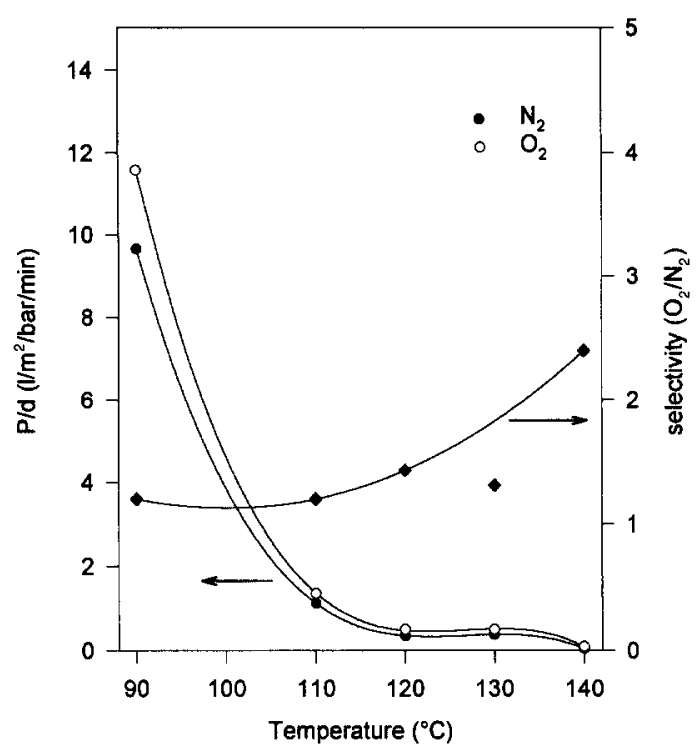

Fig. 6. Gas separation properties of the $20 \mathrm{wt} \%$ dry-spun fibres, as a function of spinneret temperature.

in an increase in skin thickness, leading to a better selectivity but a lower permeability. Another important consequence of the difference in solvent evaporation during extrusion at different spinneret temperatures, is the possibility of interference of the drying process with that of liquid-liquid demixing. This is shown schematically in Fig. 8. The possible paths through the phase diagram, experienced by the skin of the fibre, are indicated by the arrows. When the spinneret is at $150^{\circ} \mathrm{C}$, the solution vitrifies by crossing the glass transition temperature [path (a)]. At $120^{\circ} \mathrm{C}$, evaporation will cause the solution to enter the demixing domain, when it is relatively concentrated [path (b)]. Thus phase separation in the skin becomes possible, and holes can be formed. At $80^{\circ} \mathrm{C}$ the solution is completely inside the binodal, and evaporation will not have any noticeable effect on the skin properties [path (c)]. Therefore, fibres produced at lower temperatures, posses an open skin and could be used for applications other than gas separation. Even if the spinneret is at a high temperature, holes apparently can not be avoided, since the selectivity of the fibres is well below its maximum value. This is due to the lack of control of the solvent evaporation in our relatively primitive set-up.

Based upon these experiences, fibres were pro-
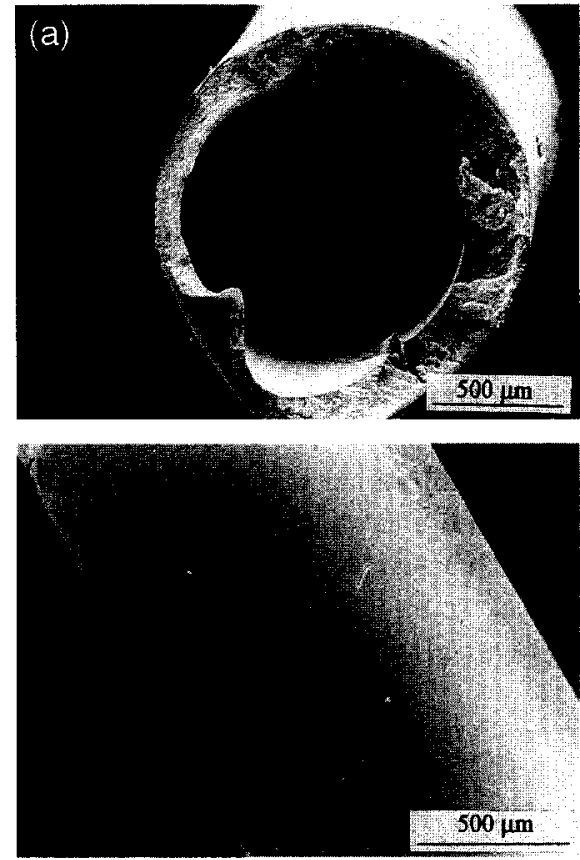

Fig. 7. Cross section (a) and side view (b) of a dry-spun $30 \mathrm{wt} \%$ fibre.

duced from more concentrated solutions. Fig. 7 shows a fibre spun with a solution of $30 \mathrm{wt} \%$ and a spinneret temperature of $130^{\circ} \mathrm{C}$. The outside of the fibre is completely smooth [Fig. 7(b)], probably due to the higher viscosity of the solution, which makes

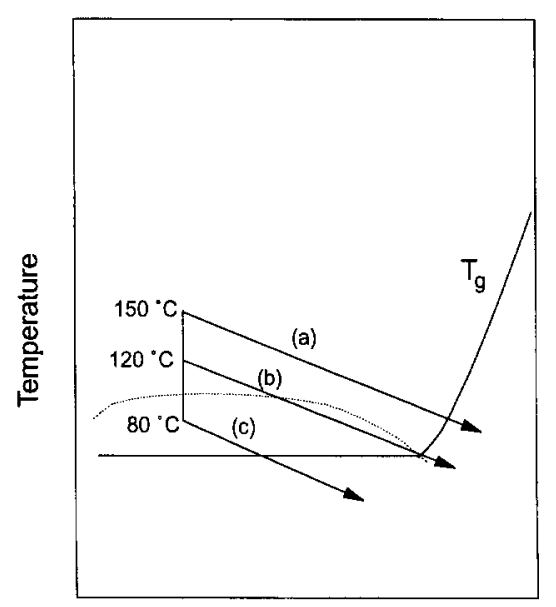

Fig. 8. Possible paths through the phase diagram, for different spinneret temperatures. 
the solution also much easier to process. Unlike the $20 \mathrm{wt} \%$ fibre this fibre did not suffer from breakage during the spinning process due to the air pressure applied on its inside. Towards the centre of the fibre, the structure of the pores is finer. The selectivity of this fibre was of the same order of magnitude as that of the $20 \mathrm{wt} \%$ fibre.

The eccentricity found in most of the fibres is not necessarily caused by a wrong alignment of the needle in the spinneret, but can be caused by a small off-axis force during haul-off. Leaving the spinneret, the solution is still rather viscous, since it takes some time to fix the morphology via vitrification. The thinner parts caused by eccentricity can be considered as weak spots that will easily break due to the internally applied air pressure.

\subsection{Air-gap spinning}

Fig. 9 shows the morphology of a fibre that was spun form a $20 \mathrm{wt} \%$ solution by the air-gap method for an air-gap length of $4 \mathrm{~cm}$ and a spinneret temperature of $140^{\circ} \mathrm{C}$. The fibre morphology is clearly asymmetric now, with pore sizes decreasing from the centre of the fibre towards its outside. The fibre has a thick smooth dense skin (Fig. 9). Close to the centre, the pores are finer than those obtained via the dry spinning process [Fig. 9(a)]. By quenching in water, cooling is much faster and this is known to result in much smaller pore diameters. Despite this, the fibre possessed poor separation qualities, since holes were still present. It proved to be difficult to run a continuous spinning process at a concentration of $20 \mathrm{wt} \%$.

In order to improve processability, the viscosity of the solution was again increased by increasing the polymer concentration to $30 \mathrm{wt} \%$. Fig. 10 shows cross sections and side views of the $30 \mathrm{wt} \%$ fibres spun with different air-gap sizes, varying from 2 to $40 \mathrm{~mm}$. The skins of all fibres are completely smooth. The cross section of the fibres obtained by this extrusion method is not always circular (Fig. 10). Despite the high cooling rates, it still takes a certain time for the solution to demix and solidify and, consequently, the fibre can still deform in the water bath. For that reason the draw-down was set such
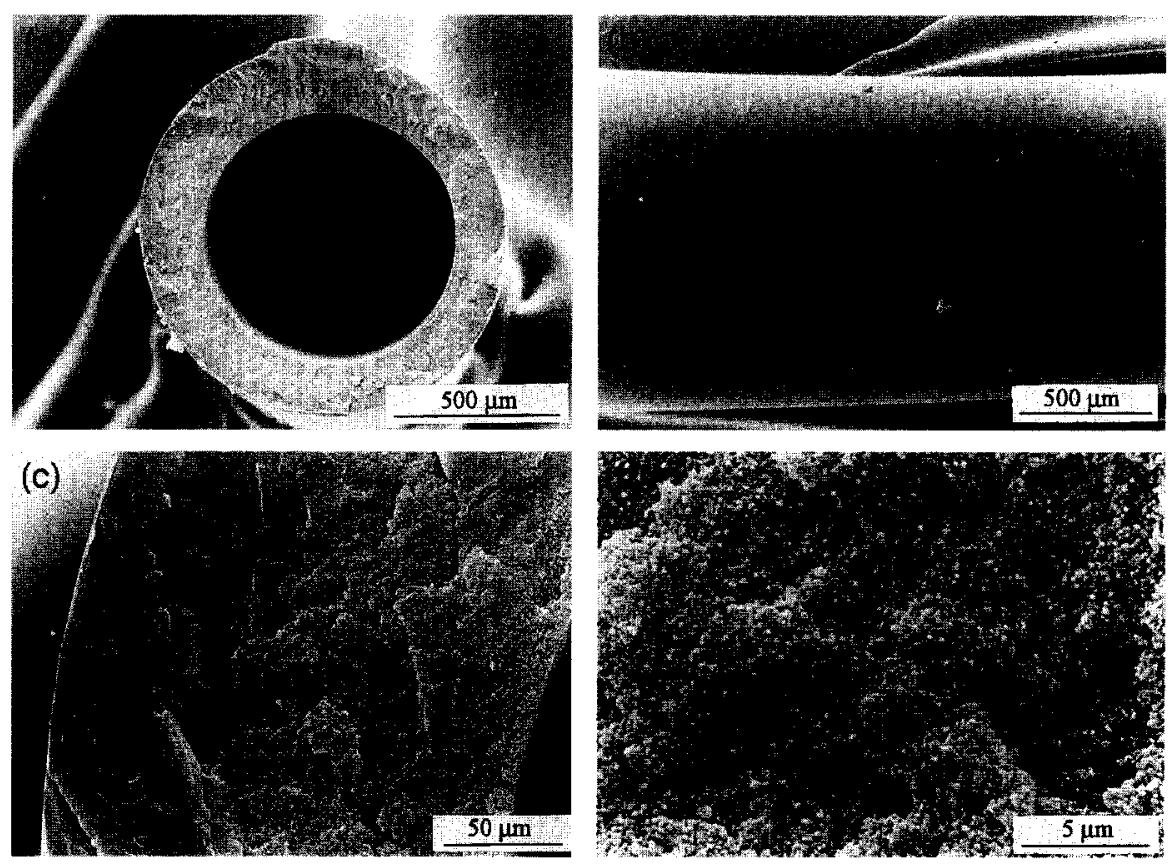

Fig. 9. Morphology of a $20 \mathrm{wt} \%$ fibre, spun with the air-gap process: (a) cross section, (b) side view, (c) fibre wall and (d) porous structure close to the centre. 
that the fibre was just under tension (haul-off values varying between 10 and $30 \mathrm{~m} / \mathrm{min}$ ).

The gas separation properties of these fibres are
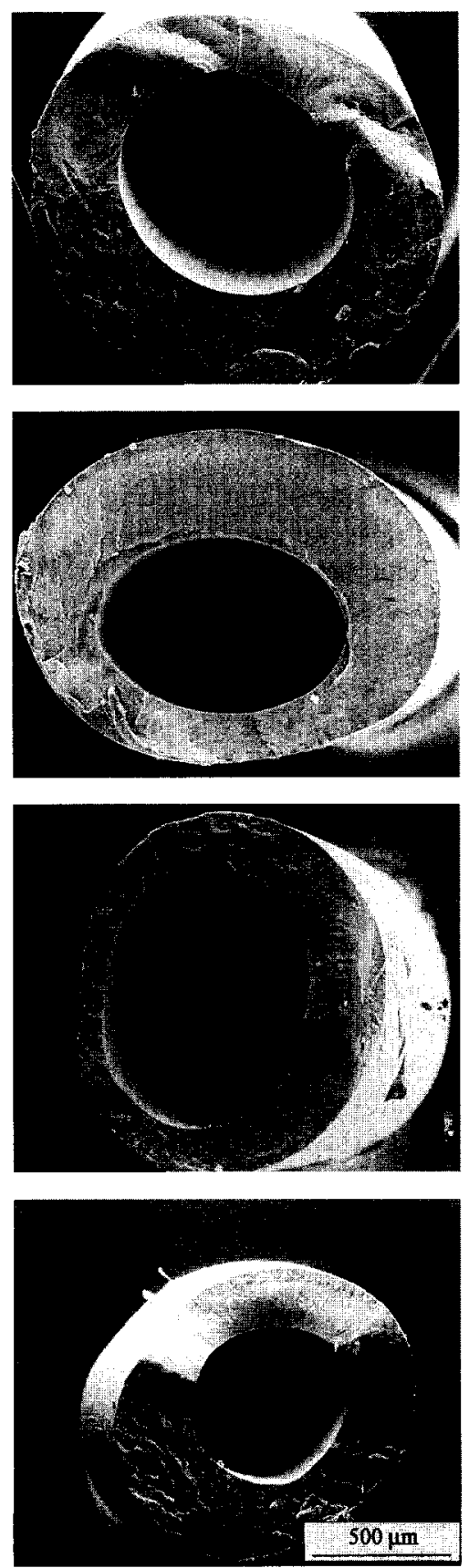

Fig. 10. Cross section and side views of $30 \mathrm{wt} \%$ fibres spun with the air-gap process, for different air-gap lengths (mm): (a) 40, (b) 20, (c) 10 and (d) 2 . (a)

(b)

(c)
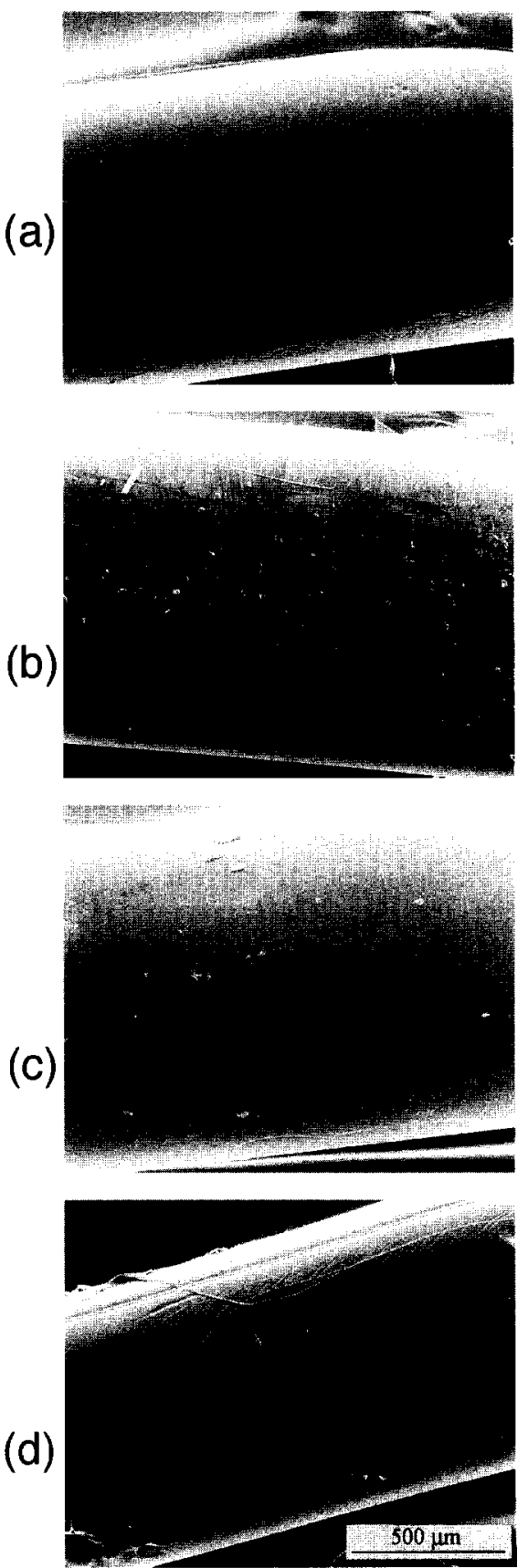

given in Fig. 11, and much better selectivities are found (between 4.5 and even 6.0). These high values are, however, partly due to the thick skins present, 


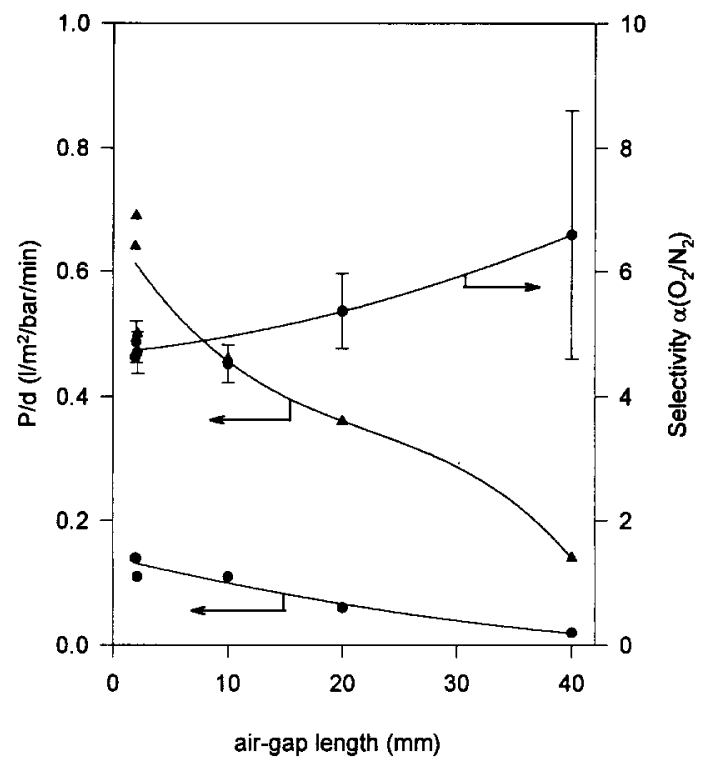

Fig. 11. Gas separation properties of the air-gap spun, $30 \mathrm{wt} \%$ fibres, as a function of the air-gap length., $\mathrm{O}_{2} ; \Delta, \mathrm{N}_{2}$.

and thus low fluxes, that of course introduce errors in the calculation of the selectivity (The larger the gap size, the thicker the skins and the larger the error). The reduced permeabilities are still low and the larger the air-gap the lower the permeability values. Apparently, evaporation of solvent in the air-gap is important for the skin formation and, consequently, for the resulting fibre properties. Since the only effect of the air-gap is a reduction in the reduced permeability, it is advisable to use a small air-gap for the production of hollow porous fibres via the TIPS process.

\section{Optimization}

\subsection{Morphologies}

In order to relate the resulting morphologies to the cooling rates applied, a calibration map was produced by preparing solutions (of $20 \mathrm{wt} \%$ PPE) at different cooling rates. The resulting morphologies are shown in Fig. 12. Below a cooling rate of $20^{\circ} \mathrm{C} / \mathrm{min}$ the solution does not only experience liquid-liquid phase separation but also crystalline structures are observed. At a cooling rate of $1^{\circ} \mathrm{C} / \mathrm{min}$ the structure is completely crystalline. This result was already found earlier, see [17]. At a cooling rate of $2.5^{\circ} \mathrm{C} / \mathrm{min}$ already some liquid-liquid phase separation has taken place in between the spherical structures. The structure is shown at two different magnifications. Up to a cooling rate of $20^{\circ} \mathrm{C} / \mathrm{min}$ a combination of liquid-liquid and solid-liquid phase separation is found. The number of spherulites and their size decreases as the cooling rate increases. Above $20^{\circ} \mathrm{C} / \mathrm{min}$, the structure is typical for liquid-liquid phase separation (at a cooling rate of $30^{\circ} \mathrm{C} / \mathrm{min}$ only very few spherulites are observed). The more the cooling rate increases, the finer the structure becomes. At the highest cooling rates the structure is almost completely closed and only some holes are present, that are, however, not interconnected. This explains why a dense skin is formed on the outside of fibres during spinning, since the cooling rates are very high there.

Optimally, this dense layer should be concentrated on the outside of the fibre only, whereas the inside of the fibre should posses an open structure, e.g. the structure at cooling rates between 50 and $380^{\circ} \mathrm{C} / \mathrm{min}$ (see Fig. 12). The cooling rate should not be too much lower than this lowest value, since in that case unwanted crystallization could be induced. When the porous structure of the dry spun fibres is compared with the calibration standard in Fig. 12, apparently a cooling rate was experienced around $150^{\circ} \mathrm{C} / \mathrm{min}$. For the case of the air-gap spun fibres, the pore size on the inside is much smaller than for the dry spinning process. Apparently, the experienced cooling rate varied from about $21000^{\circ} \mathrm{C} / \mathrm{min}$, on the inside, to 57600 , on the outside of the fibres.

In order to obtain an ideal structure it seems necessary to use a combination of dry and air-gap spinning. It is expected that by cooling a fibre a short time in an effective cooling medium like water, the outside of the fibre will experience a high cooling rate, whereby subsequent transport of the fibre through the open air will result in slower cooling and consequently a more open structure on the inside of the fibre will be obtained. In order to check this, some elementary calculations were performed. Phase separation is not taken into account and the thermal properties of the fibre and of the solutions are taken constant. These assumptions are reasonable as long 
as the solution does not vitrify, since the thermal properties of the solid continuous polymer in the presence of a solvent are completely different from those of its solution. Given all these simplifications, it is not expected that this approach yields more than a first idea only of the effect of different cooling histories on the final fibre morphology.

\subsection{Modelling calculations of the non-isothermal solution spinning}

First the necessary equations are summarized. We try to calculate the approached temperature profile in the fibre as a function of time. In first instance, it is assumed that no draw-down or gravity is exerted on the fibre and that inertia can be neglected in these typically low Reynolds number flows. Therefore the fibres moves at a constant axial and radial velocity. With these simplifying assumptions, the momentum equation need to be solved and we can focus on the energy equation. When a solution is cooled in the open air, evaporation of solvent can be important and the relevant equation of continuity can be written as (in cylindrical coordinates):

$$
\frac{\partial \phi_{\mathrm{s}}(r)}{\partial t}=D_{\mathrm{PS}}\left(\frac{1}{r} \frac{\partial \phi_{\mathrm{s}}(r)}{\partial r}+\frac{\partial^{2} \phi_{\mathrm{s}}(r)}{\partial r^{2}}\right)
$$

where $\phi_{\mathrm{s}}$ is the solvent volume fraction and $D_{\mathrm{PS}}$ is the diffusion coefficient of polymer and solvent. On the inside of the hollow fibre, it is assumed that no evaporation takes place [Eq. (5)] while on its outside the diffusion of solvent out of the fibre equals the mass transferred by evaporation [Eq. (6)]:

$$
\begin{aligned}
& \left.\frac{\partial \phi_{\mathrm{s}}(r)}{\partial r}\right|_{R_{1}}=0 \\
& \left.D_{\mathrm{PS}} \frac{\partial \phi_{\mathrm{s}}(r)}{\partial r}\right|_{R_{2}}+k_{\mathrm{x}}\left(\phi_{\mathrm{so}}\left(R_{2}\right)-\phi_{\mathrm{s}}(\infty)\right)=0
\end{aligned}
$$

where $k_{\mathrm{x}}$ is the mass transfer coefficient on the outside of the membrane, $R_{1}$ is the inner radius of the hollow fibre and $R_{2}$ the outer radius. In Eq. (6), $\phi_{\mathrm{so}}\left(R_{2}\right)$ is the concentration on the air-side of the interface. Normally, the concentration at the interface on the inside of the membrane is proportional to $\phi_{\mathrm{so}}\left(R_{2}\right)$, with a factor determined by the vapor pressure of the solvent and the thermodynamic parameters of the polymer-solvent system [24-26]. As an approximation, both concentrations are assumed to be equal.

For the temperature, analogous expressions as Eqs. (4)-(6) can be written. As a first approximation, the temperature change in time is due to radial conductivity only:

$\rho C_{\mathrm{P}} \frac{\partial T(r)}{\partial t}=k\left(\frac{1}{r} \frac{\partial T(r)}{\partial r}+\frac{\partial^{2} T(r)}{\partial r^{2}}\right)$

where $\rho$ is the density of the polymer solution, $C_{\mathrm{P}}$ is the heat capacity, and $k$ is the thermal conductivity. The boundary conditions read:

$$
\begin{aligned}
& \left.\frac{\partial T(r)}{\partial r}\right|_{R_{1}}=0 \\
& \left.k \frac{\partial T(r)}{\partial r}\right|_{R_{2}}+h\left(T\left(R_{2}\right)-T(\infty)\right)+\Delta H k_{\mathrm{x}}\left(\phi_{\mathrm{so}}\left(R_{2}\right)\right. \\
& \left.-\phi_{\mathrm{s}}(\infty)\right)=0
\end{aligned}
$$

where $h$ is the heat transfer coefficient of the surrounding medium, $\Delta H$ is the heat of vaporization of the solvent. A set of two coupled equations results. In order to simplify the analysis, it is assumed that all parameters such as the diffusion coefficient, heat capacity, thermal conductivity and density are temperature independent. This makes it possible to solve Eq. (4) first and solve Eq. (7) for the temperature next. If the fibre is cooled in water, evaporation is neglected.

Of course, the material and process parameters need to be known beforehand. For most parameters, the values for pure cyclohexanol were used, since the values for PPE-cyclohexanol are not known. For the determination of $h$, different expressions exist $[23,25,26]$ and $h$ is related to $k$ via the Nusselt number $(N u)$ :

$h=\frac{N u k}{D}$

where $D$ is the fibre diameter. The Nusselt number for fibres, cooled by moving them in a stagnant medium, can be written as $[25,26]$ :

$N u=0.35+0.146 R e^{0.50}$

The Reynolds number is defined as:

$R e=\frac{\rho v D}{\mu}$ 

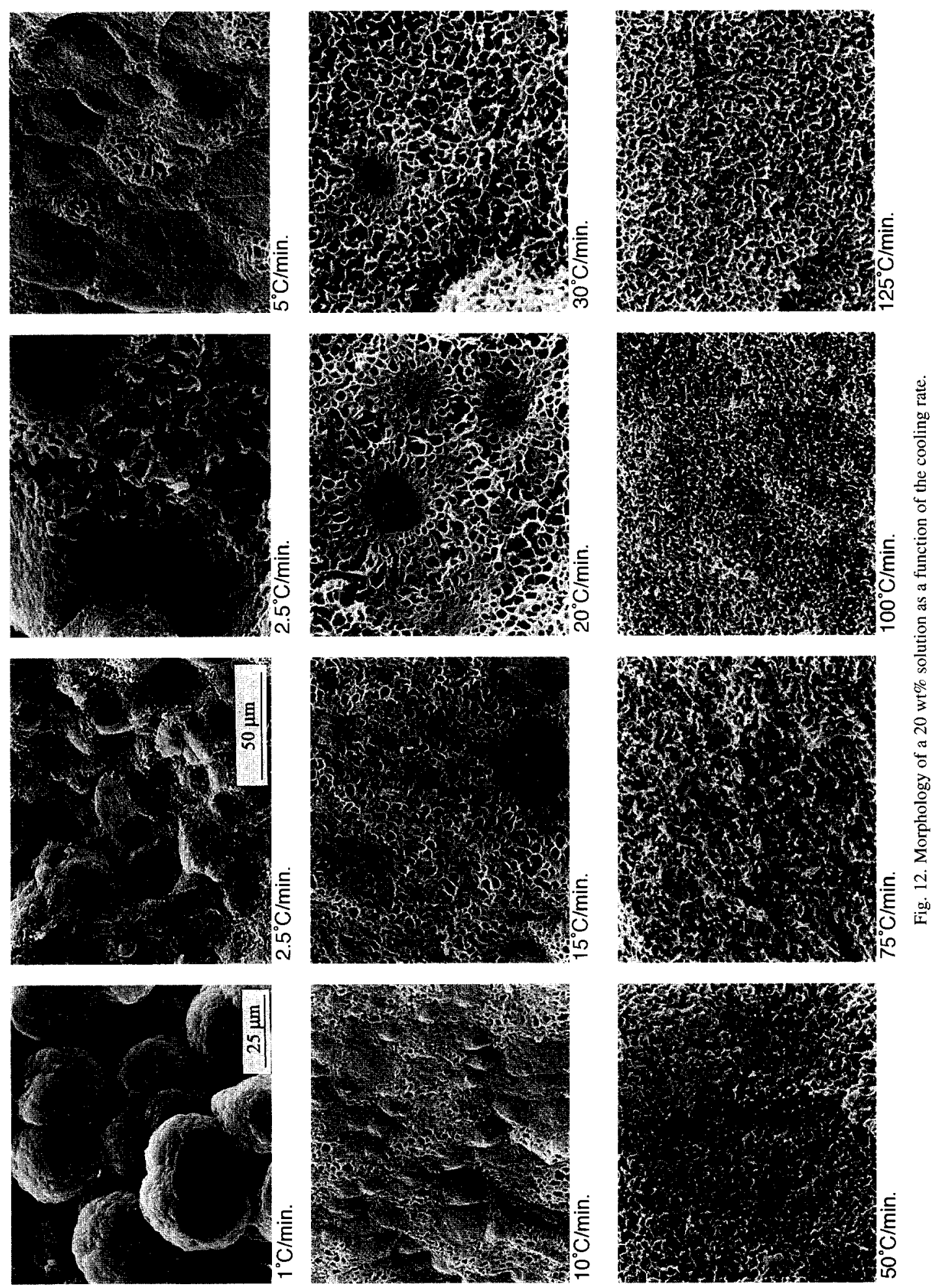

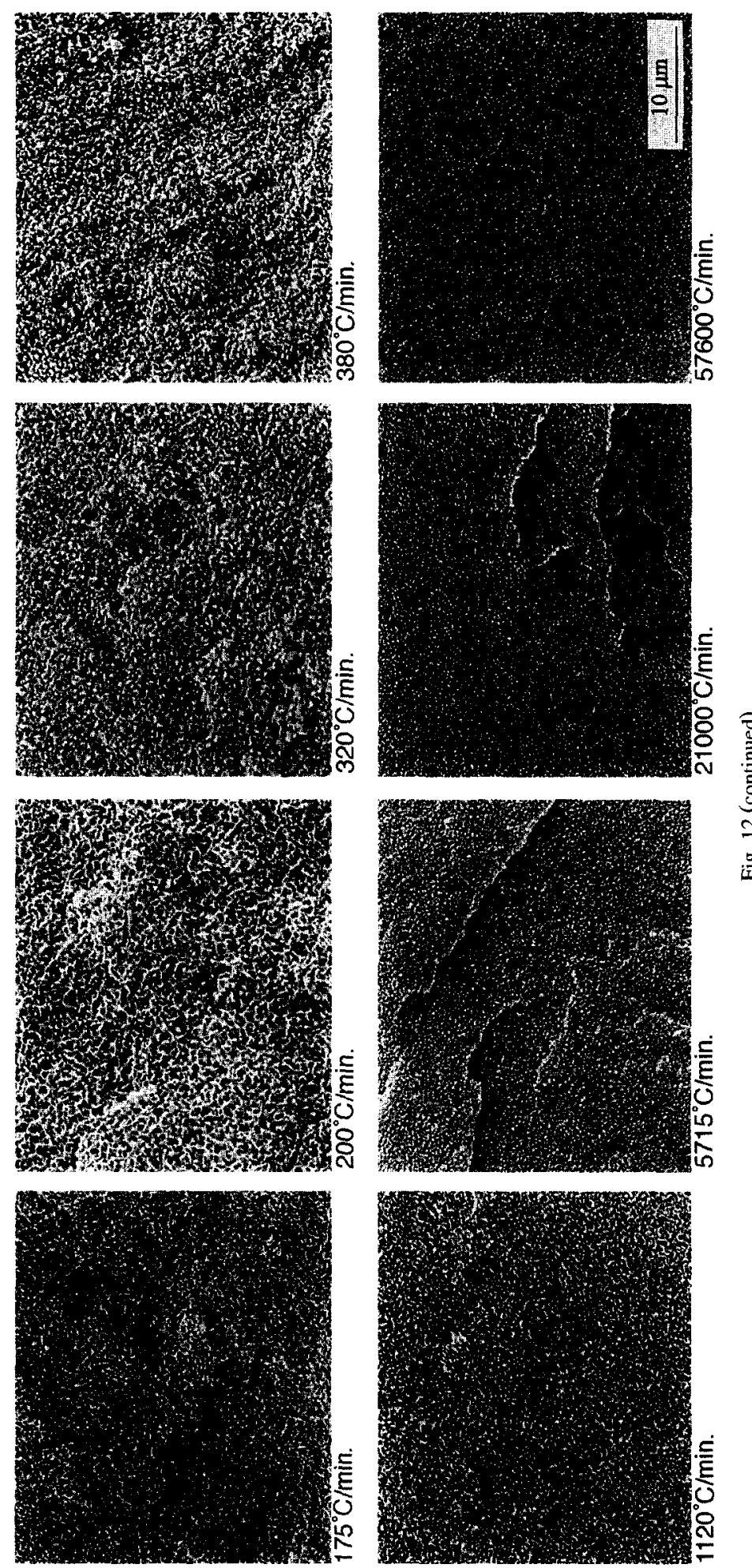
where $\rho$ is the density of the surrounding medium, $v$ is the velocity at which the fibre moves and $\mu$ is the viscosity of the surrounding medium. Once $h$ is known, $k_{\mathrm{x}}$ can be estimated, since in a first approximation $h$ and $k_{\mathrm{x}}$ are proportional. Ohzawa et al. [26] determined their ratio for some solvents. From their data we took a value of $7.5 \mathrm{cal} /{ }^{\circ} \mathrm{C} \mathrm{mol}$, which is the mean value for different temperatures for dimethylformamide (DMF), a solvent with a high boiling point $\left(153^{\circ} \mathrm{C}\right)$ like cyclohexanol $\left(161^{\circ} \mathrm{C}\right)$ :

$\frac{h}{k_{\mathrm{x}}}=7.5 \frac{\mathrm{cal}}{{ }^{\circ} \mathrm{C} \mathrm{mol}}=31.2 \frac{\mathrm{J}}{{ }^{\circ} \mathrm{C} \mathrm{mol}}$

$D_{\mathrm{PS}}$ for PPE-cyclohexanol is not known. In [27], the diffusion coefficient for the system PPE-trichloroethylene (TCE) has been determined and was taken to be concentration dependent. Here we will use a constant diffusion coefficient. It is to be expected that diffusion of PPE in TCE will go much easier than in cyclohexanol, since TCE is a much better solvent for PPE, so $D_{\mathrm{PS}}$ is an upper limit. All parameters used in the calculations are summarized in Table 1. Eqs. (4) and (7) are both solved numerically using an implicit, stable, Cranck-Nicholson scheme. For this purpose, the membrane is divided into 11 layers.

Table 1

Parameters used in the calculations

\begin{tabular}{lc}
\hline$\phi_{\mathrm{s}}(\mathrm{vol} \%), t=0$ & 81.3 \\
$\phi_{\mathrm{s}}(\infty)(\mathrm{vol} \%)$ & 0.0 \\
$T\left({ }^{\circ} \mathrm{C}\right), t=0$ & 140 \\
$v(\mathrm{~m} / \mathrm{s})$ & 0.1667 \\
$D(\mathrm{~mm})$ & 1.0 \\
Thickness $(\mu \mathrm{m})$ & 200.0 \\
$k($ cyclohexanol $)(\mathrm{W} / \mathrm{m} \mathrm{K})$ & 0.161 \\
$k($ water $)(\mathrm{W} / \mathrm{m} \mathrm{K})$ & 0.614 \\
$k($ air $)(\mathrm{W} / \mathrm{m} \mathrm{K})$ & 0.0262 \\
$\rho($ polymer solution $)\left(\mathrm{kg} / \mathrm{m}^{3}\right)$ & 985.4 \\
$\rho($ water $)\left(\mathrm{kg} / \mathrm{m}^{3}\right)$ & 1000.0 \\
$\rho($ air $)\left(\mathrm{kg} / \mathrm{m}^{3}\right)$ & 1.2928 \\
$\mu \times 10^{-4}(\mathrm{water})(\mathrm{Pa} \mathrm{s})$ & 8.57 \\
$\mu \times 10^{-5}($ air $)(\mathrm{Pa} \mathrm{s})$ & 2.0 \\
$C_{\mathrm{p}}(\mathrm{cyclohexanol})\left(\mathrm{kJ} / \mathrm{kg}{ }^{\circ} \mathrm{C}\right)$ & 1.739 \\
$h($ water $)\left(\mathrm{W} / \mathrm{m}^{2} \mathrm{~K}\right)$ & 1450.0 \\
$h($ air $)\left(\mathrm{W} / \mathrm{m}^{2} \mathrm{~K}\right)$ & 23.0 \\
$k_{\mathrm{x}} \times 10^{-5}($ air $)(\mathrm{m} / \mathrm{s})$ & 7.19 \\
$D_{\mathrm{PS}} \times 10^{-11}\left(\mathrm{~m}^{2} / \mathrm{s}\right)$ & 1.38 \\
$\Delta H(\mathrm{~J} / \mathrm{kg})$ & 449280.0 \\
\hline
\end{tabular}

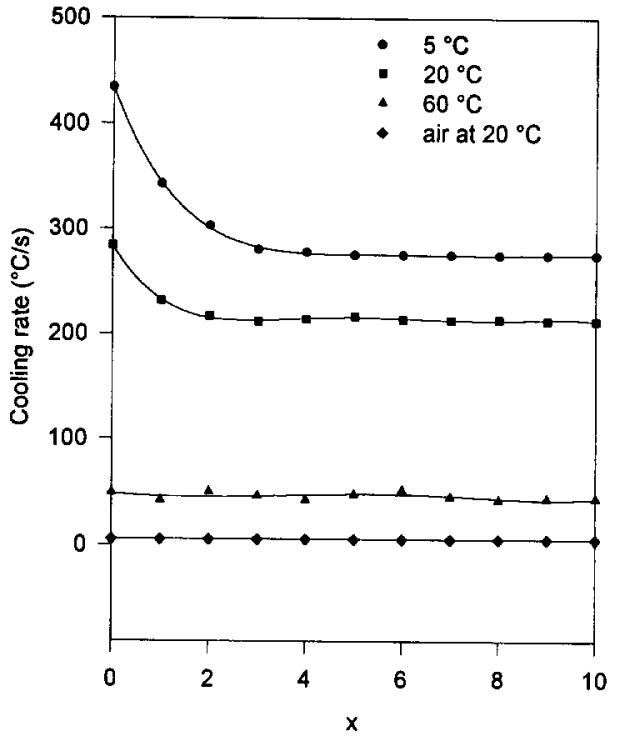

Fig. 13. Cooling rates at $70^{\circ} \mathrm{C}$ as a function of fibre thickness $(x)$, when cooled in water at different temperatures, and air at $20^{\circ} \mathrm{C}$.

With the aid of the above, simple model, we will now try to find a way for improving the fibre properties, by optimizing the spinning process. Fig. 13 shows the calculated cooling rates at $70^{\circ} \mathrm{C}$ for fibres cooled in water of different temperatures and

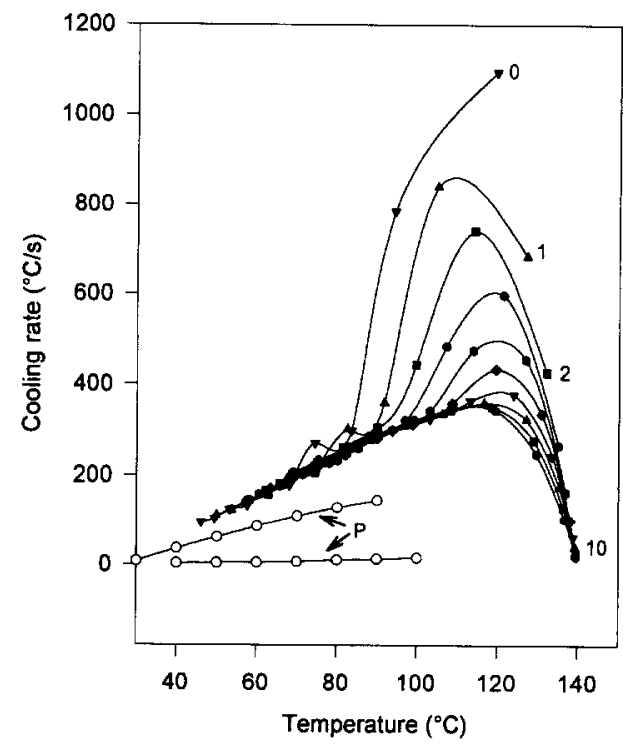

Fig. 14. Cooling rate-temperature relationship for the different layers in the membrane, the "Palermo"-curves are labeled with P. 
in air (at $20^{\circ} \mathrm{C}$ ), across the thickness of the fibre $(x)$. When the fibre is cooled in air, the cooling rate is flat, which is in agreement with the uniformly porous structure, obtained via dry spinning. When cooling fibres in water of $20^{\circ} \mathrm{C}$, the cooling rate is more asymmetric, with a value of about $300^{\circ} \mathrm{C} / \mathrm{s}$ $\left(18000^{\circ} \mathrm{C} / \mathrm{min}\right)$ on the outside, and about $200^{\circ} \mathrm{C} / \mathrm{s}$ on the inside $\left(12000^{\circ} \mathrm{C} / \mathrm{min}\right)$. This is not as asym- metric as we would have expected, from comparing the resulting experimental fibre structure (Fig. 9) with the calibration morphologies in Fig. 12. To solve this discrepancy one should, however, remind that it is to be expected that the cooling rate at which the demixing domain is entered (at about $100^{\circ} \mathrm{C}$ ) also influences the structures formed (and not only the cooling rate at $70^{\circ} \mathrm{C}$ as taken here to be representa-
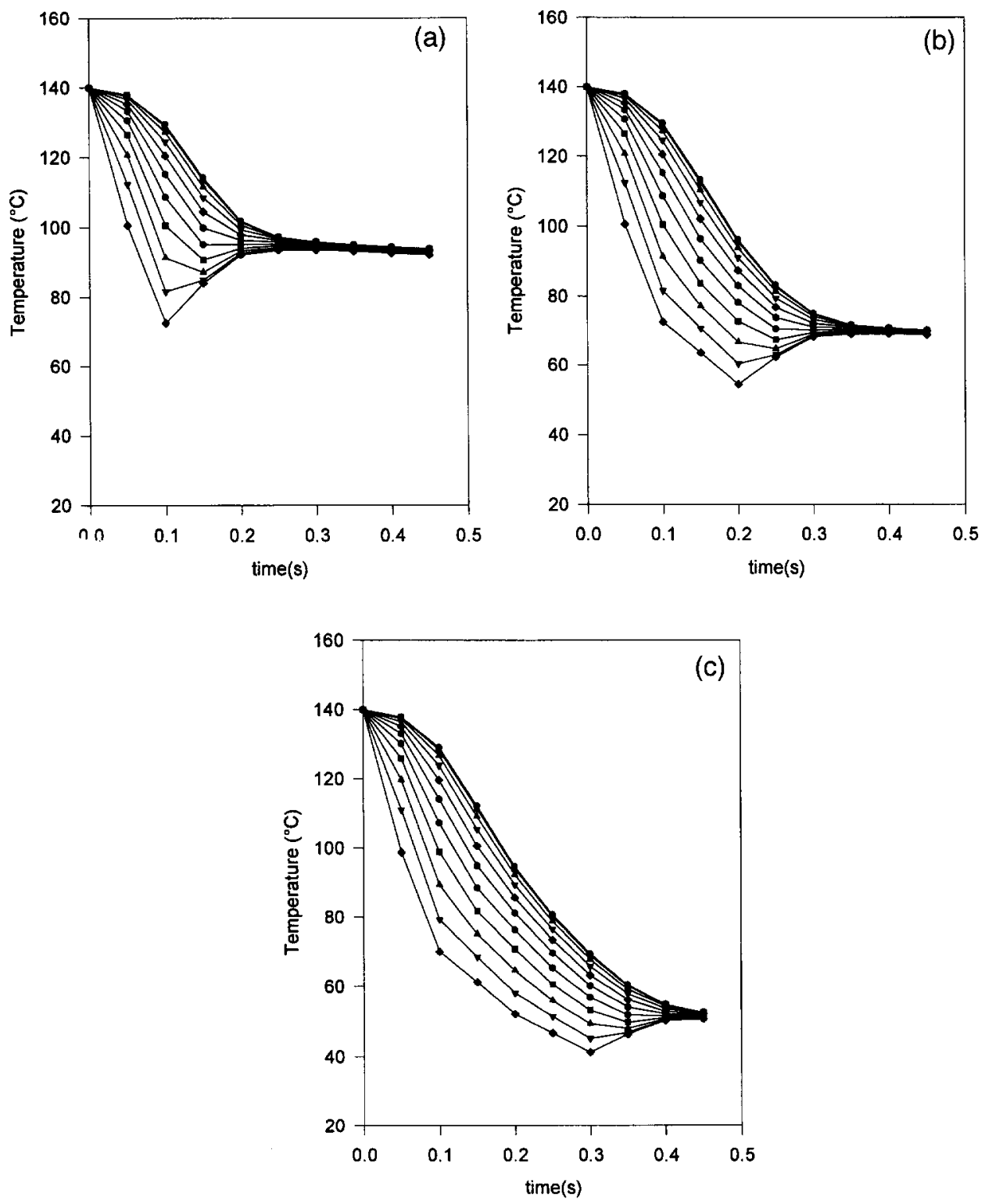

Fig. 15. Temperature change for different residence times in the water bath: (a) $0.1 \mathrm{~s}$, (b) $0.2 \mathrm{~s}$ and (c) $0.3 \mathrm{~s}$. 
tive). Fig. 14 shows the calculated cooling rate-temperature relationship for the different layers. In order not to overload the figure, not every curve has been labelled. Two measured curves for the Palermo apparatus are included, for which these cooling curves are linear. In the fibre, the outside layers $(0,1,2, \ldots)$ have a completely different thermal history than the inside layers $(. ., 8,9,10)$. This means that even if at $70^{\circ} \mathrm{C}$ the same cooling rate is reached in the different layers, the cooling rate history is different and most likely a different structure is obtained. Therefore the calculations not necessarily underpredict the cooling rate, but care has to be taken to compare a structure at a cooling rate at $70^{\circ} \mathrm{C}$ with a membrane structure at the same cooling rate, without considering the previous thermal history of the membrane. Lowering the temperature of the cooling medium $\left(5^{\circ} \mathrm{C}\right)$ will make the cooling rate curve more asymmetric, but also increases the overall cooling rate, thereby making the structure on the inside also denser. Increasing the temperature to $60^{\circ} \mathrm{C}$ of the cooling medium will lower the cooling rate significantly, but now the cooling rate curve becomes much flatter, which is unfavourable for the formation of a dense skin.

Another way for altering the cooling rate history, is the use of both water and air as cooling media. If the fibre is cooled in water for a short time and then allowed to cool in air, it is possible to get a high cooling rate on the outside, combined with a low cooling rate on the inside. Fig. 15 shows the temperature change in time for different residence times in the water bath. If the fibre only stay for $0.1 \mathrm{~s}$ in water [Fig. 15 (a)] (this is a length of $16 \mathrm{~mm}$, at the spinning velocities used), the outside of the fibre will be cooled rapidly, while as soon as the fibre leaves the water it will be heated by the inside layers of the fibre and when the fibre finally reaches $70^{\circ} \mathrm{C}$, the cooling rate is both low and flat, which is unfavourable (Fig. 16). However, when the fibre is kept $0.2 \mathrm{~s}$ in water $(32 \mathrm{~mm})$, the outside is cooled rapidly again, but now to a temperature below $70^{\circ} \mathrm{C}$ thereby creating a dense skin. The inside is cooled much slower, and now does not reheat the outside to above $70^{\circ} \mathrm{C}$ [Fig. 15 (b)]. Now a low cooling rate (at $70^{\circ} \mathrm{C}$ ) is obtained in the inside of the fibre, combined with the high cooling rate on the outside (Fig. 16). This implies that a much more open structure is formed on the inside. Leaving the fibre $0.3 \mathrm{~s}$ in water, cools all layers to below $70^{\circ} \mathrm{C}$ before they experience cooling in the air [Fig. 15 (c)], so in fact the cooling process is over. In order to test the hypothesis, it was tried to spin fibres applying different residence times in the water bath.

\subsection{Improved fibre spinning}

Only some preliminary experiments were carried out using a solution of $30 \mathrm{wt} \%$ of PPE in cyclohexanol. The take-up speed was set at $20 \mathrm{~m} / \mathrm{min}$ and the air-gap at $10 \mathrm{~mm}$. This value of the take-up speed is higher than the one used in the calculations, but for the $30 \mathrm{wt} \%$ fibres a lower speed is not effective, since then the fibre is not drawn down enough to be put under sufficient tension. The residence time in the bath was controlled by adjusting the depth of the roll around which the fibre is pulled [see Fig. 3(b)]. Three different residence times were used: $0.15,0.30$ and $0.45 \mathrm{~s}$. The resulting different porous structures are shown as a function of the residence time in water in Fig. 17. For short times $(0.15 \mathrm{~s})$ the structure is more open while for the longer times $(0.30-0.45 \mathrm{~s})$, the structure is found to be comparable to the one found in normal air-gap spinning. However, the differences found can not be

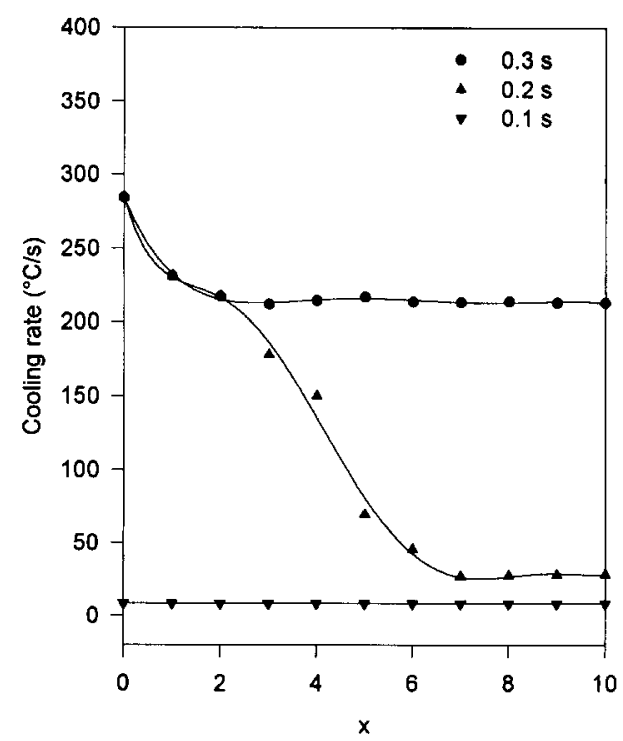

Fig. 16. Cooling rates at $70^{\circ} \mathrm{C}$ for the different layers $(x)$ as a function of the residence time in the bath. 
Table 2

Permeability and selectivity for oxygen and nitrogen for different residence times in the water bath

\begin{tabular}{llll}
\hline $\begin{array}{l}\text { Residence } \\
\text { time }(\mathrm{s})\end{array}$ & $\begin{array}{l}P / d\left(\mathrm{O}_{2}\right) \\
\left(1 / \mathrm{m}^{2} / \mathrm{bar} / \mathrm{min}\right)\end{array}$ & $\begin{array}{l}P / d\left(\mathrm{~N}_{2}\right) \\
\left(1 / \mathrm{m}^{2} / \mathrm{bar} / \mathrm{min}\right)\end{array}$ & $\alpha_{\mathrm{O}_{2} / \mathrm{N}_{2}}$ \\
\hline 0.15 & 0.10 & 0.022 & 4.6 \\
0.30 & 0.11 & 0.022 & 4.9 \\
0.45 & 0.09 & 0.020 & 4.5 \\
\hline
\end{tabular}

considered as very spectacular. As stated, these experiments were only preliminary and serve as a first indication. In conclusion, cooling to the point at which the structure vitrifies, proceeds rapidly $(0.3-$ $0.45 \mathrm{~s}$ ), as predicted by the model.

Table 2 shows the gas separation properties of these fibres. No real influence of the residence time is noticeable. After $0.15 \mathrm{~s}$, a fibre is obtained with good gas separation properties, but still a too thick skin. The porous structure underneath the skin is more open, but apparently this does not improve the flux through the membrane. So even though the porous structure of the membrane seems much denser for a residence time of $0.5 \mathrm{~s}$, it is as open to gas flow as the structure formed at a residence time of only $0.15 \mathrm{~s}$. This proves that the resistance towards the gas flow is concentrated at the surface of the membrane, and more effective methods for producing a thinner skin by cooling have to be found. We did not test the effect of residence times below $0.15 \mathrm{~s}$, since this was not realizable with our spinning apparatus. This might be worth trying.

\section{Conclusions}

It is shown that when thermal phase separation is used, membranes with a closed skin can be obtained. With dry spinning, a thin skin is obtained on the outside of the fibre formed by evaporation of the solvent, which is easy, since the dissolution temperature $\left(150^{\circ} \mathrm{C}\right)$ is close to the boiling temperature of the solvent $\left(16^{\circ} \mathrm{C}\right)$. By lowering the temperature of
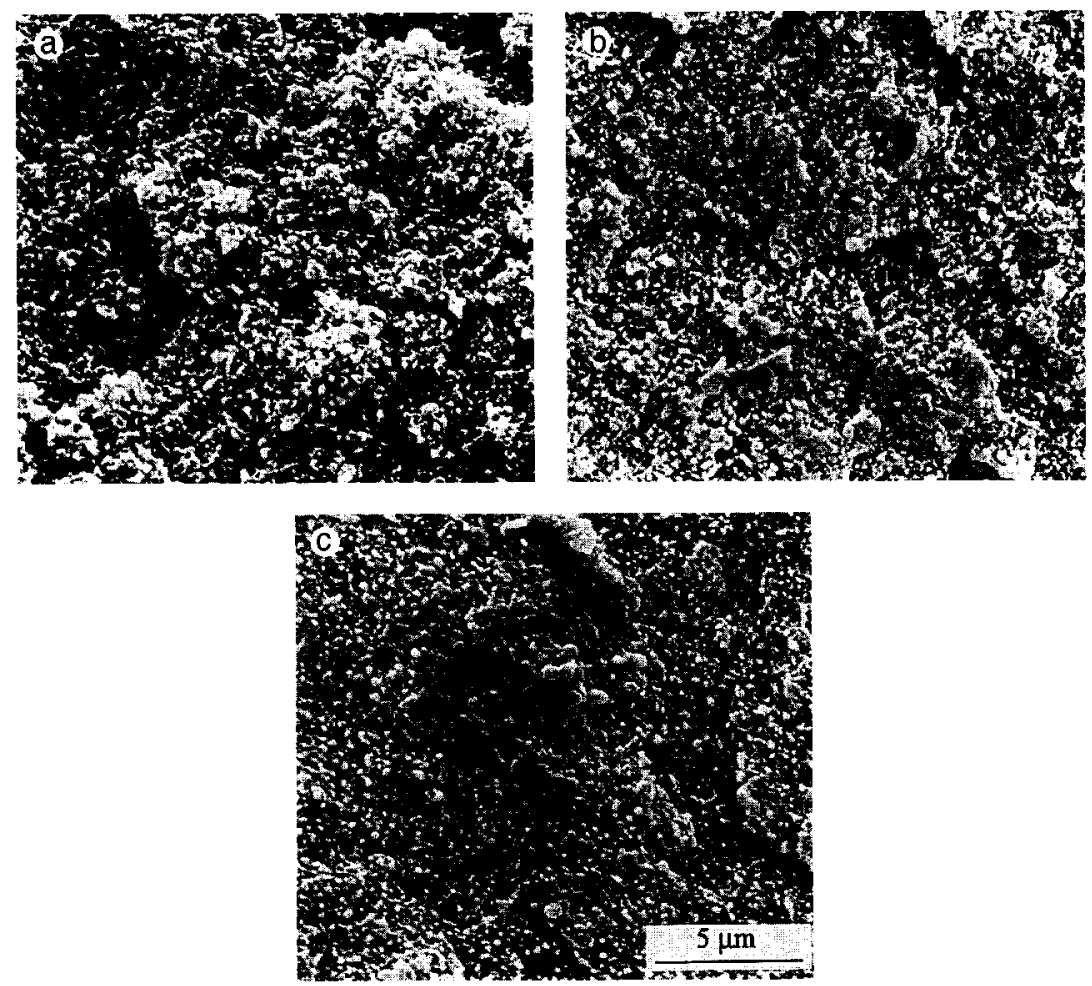

Fig. 17. Membrane structure for different residence times in the bath: (a) $0.15 \mathrm{~s}$, (b) $0.30 \mathrm{~s}$ and (c) $0.45 \mathrm{~s}$, PPE (30 wt\%) -cyclohexanol. 
the spinneret, fibres with an open skin can be produced which are, however, not suited for gas separation. Moreover, care has to be taken not to lower the temperature too much, since then solidification inside the spinneret occurs, leading to blockage.

The air-gap method results in the formation of a highly asymmetric porous structure. By rapid cooling in water, a steep temperature gradient is established across the fibre cross section, producing a gradient in the structure of the fibre. These fibres posses a closed outer skin given the locally high cooling rates. They can be used to separate oxygen and nitrogen, however given the too thick skins, the fluxes are still too low. By decreasing the air-gap, the reduced permeability increases somewhat, whereas the selectivity decreases. Small air-gaps are therefore preferable.

Analysis of morphologies produced under controlled circumstances creates a calibration tool which reveals that, in the case of dry fibre spinning, cooling rates of about $150^{\circ} \mathrm{C} / \mathrm{min}$ are achieved, whereas in the case of air-gap spinning the cooling rates vary from $21000^{\circ} \mathrm{C} / \mathrm{min}$ on the inside to more than $57600^{\circ} \mathrm{C} / \mathrm{min}$ on the outside. These high cooling rates produce dense skins that are still too thick. Ideally, high cooling rates should be concentrated on the outside of the membrane only.

The cooling rate history of the samples can approximately be calculated with a simple model. An attempt was made to calculate more optimal spinning conditions and the simulations showed that fibres with a more open porous structure combined with a closed skin can be produced by cooling them for only a short time in water, followed by cooling in air. Fibres were spun approximating these conditions, and a more open porous structure was obtained, however no change in gas separation properties was measured. The skin thickness apparently is not altered enough, and other more efficient cooling techniques have to be developed in order to produce thin enough skins. Also it might be interesting to look at other solvents (or solvent-combinations) or other polymers, that behave differently upon cooling. For this purpose, modelling will be a useful tool, since it is possible to look at the effect of different thermal properties of the solvent and the polymer or different heat transfer coefficients on the resulting cooling history of the fibre.

\section{Acknowledgements}

The authors wish to thank the National Fund for Scientific Research (N.F.W.O.) and T.N.O. Delft for their financial support.

\section{References}

[1] S. Loeb and S. Sourirajan, Sea water demineralization by means of an osmotic membrane, Adv. Chem. Ser., 38 (1962) 117.

[2] M. Mulder, in Basic principles of membrane technology, Kluwer Academic Publishers, Dordrecht, The Netherlands, 1991.

[3] A.J. Castro, Method for making microporous products, US Pat., 4,247,498 assigned to Akzona Inc., 1980.

[4] G.H. Vitzhum and M.A. Davis, 0.1 Micron rated polypropylene membrane and method for its preparation, US Pat., 4,490,431 assigned to Akzona Inc., 1984.

[5] G.H. Shipman, Microporous sheet materials, method of making and articles made therewith, US Pat., 4,539,256 assigned to $3 \mathrm{M}$ Company, 1984.

[6] R.E. Kesting, Phase inversion membranes, ACS Symp. Ser., 269 (1985) 131.

[7] W.C. Hiatt, G.H. Vitzhum, K.B. Wagener, K. Gerlach and C. Josefiak, Microporous membranes via upper critical temperature phase separation, ACS Symp. Ser., 269 (1985) 229.

[8] G.T. Caneba and D.S. Soong, Polymer membrane formation through the thermal-inversion process, 1. Experimental study of the membrane structure formation, Macromolecules, 18 (1985) 2538.

[9] G.T. Caneba and D.S. Soong, Polymer membrane formation through the thermal-inversion process, 2. Mathematical modeling of the membrane structure formation, Macromolecules, 18 (1985) 2545.

[10] D.R. Lloyd and J.W. Barlow, Microporous membrane formation via thermally-induced phase separation, AIChE Symp. Ser., 261 (1988) 28.

[11] D.R. Lloyd, S.S. Kim and K.E. Kinzer, Microporous membrane formation via thermally-induced phase separation, II. Liquid-liquid phase separation, J. Membrane Sci., 64 (1991) 1.

[12] P. Vandeweerdt, H. Berghmans and Y. Tervoort, Temperature-concentration behaviour of solutions of polydisperse, atactic poly(methylmethacrylate) and its influence on the formation of amorphous, microporous membranes, Macromolecules, 24 (1991) 3547.

[13] S.S. Kim and D.R. Lloyd, Microporous membrane formation via thermally-induced phase separation. III. Effect of thermodynamic interactions on the structure of isotactic polypropylene membranes, J. Membrane Sci., 64 (1991) 13.

[14] L. Aerts, M. Kunz, H. Berghmans and R. Koningsveld, Relation between phase behaviour and morphology in 
polyethylene-diphenylether systems, Makromol. Chem., 194 (1993) 2697.

[15] J. Arnauts, H. Berghmans and R. Koningsveld, Structure formation in solutions of atactic polystyrene in trans-decalin, Makromol. Chem., 194 (1993) 77.

[16] H. Berghmans and S. Berghmans, Structure formation in polymeric systems by solution demixing, Europhys. Conf. Abstr., 18C (1994) L2-1.

[17] S. Berghmans, H. Berghmans, H. Meijer and J. Mewis, Phase behaviour and structure formation in solutions of poly(2,6-dimethyl-1,4-phenylene ether) in cyclohexanol, Polymer, 36 (1995) 3085.

[18] S. Berghmans, H. Berghmans, H. Meijer and J. Mewis, Structure formation during spinning of hollow porous fibres from solutions of poly(2,6-dimethyl-1,4-phenylene ether) in cyclohexanol, Abstr. 11th Ann. Meeting PPS, Seoul, Korea, 1995.

[19] T. Graham, Philos. Mag., 32 (1866) 401.

[20] C.J.M. Bauer, J. Smid, J. Olijslager and C.A. Smolders, The resistance towards gas transport of the sublayer of asymmetric PPO hollow fibre membranes determined by plasma etching, J. Membrane Sci., 57 (1991) 307.
[21] V. Brucato, G. Crippa, S. Piccarolo and G. Titlomanio, Crystallization of polymer melts under fast cooling. I. Nucleated polyamide 6, Polym. Eng. Sci., 31 (1991) 1411.

[22] S. Piccarolo, M. Saiu, V. Brucato and G. Titomanlio, Crystallization of polymer melts under fast cooling. II. High-purity iPP, J. Appl. Polym. Sci., 46 (1992) 625.

[23] A. Ziabicki, in Fundamentals of Fibre Formation, Wiley, New York, 1976.

[24] I. Sakurada, in Polyvinyl Alcohol Fibers, Marcel Dekker, New York, 1985.

[25] S.S. Sohaje, W.B. Krantz and A.R. Greenberg, Dense polymer film and membrane formation via the dry-cast process, Part I. Model development, J Membrane Sci., 94 (1994) 281.

[26] Y. Ohzawa, Y. Nagano and T. Matsuo, Studies on dry spinning. I. Fundamental equations, J. Appl. Polym. Sci, 13 (1969) 257.

[27] Y. Ohzawa and Y. Nagano, Studies on dry spinning, II. Numerical solutions for some polymer-solvent systems, based on the assumption that drying is controlled, J. Appl. Polym. Sci., 14 (1970) 1879. 